\title{
Analysis and Interpretation of Coronary Artery Blockage using Soft Computing Techniques
}

\author{
Ankur Sharma ${ }^{1}$, Dr.Saurabh Mukherjee ${ }^{2}$ \\ Research Scholor ${ }^{1}$, Associate Professor ${ }^{2}$, Banasthali University
}

\begin{abstract}
Analysis and interpretation of Myocardial infarction, is very important at the early stage to detect the possible risk for heart attacks. Various non-invasive imaging modalities have been used for better diagnosis. Cardiologist wants better image resolution and detailed descriptions of the ROI (Region of Interest). In the present paper, the images have been pre-processed for further applications like filtering, denoising and deblurring by using various techniques like the usages of average and median filter, soft thresholding for various segmentation coronary blockages. The vessel segmentation taken from CT angiogram DICOM images. The vessel segmentation X-Ray image has been processed by fuzzy system. The gradient of the image has been taken after the conversion into double precision. 24 different 'If Then' rules have been initiated and the defuzzification method has been done by centriod method.
\end{abstract}

\section{Introduction}

Cardiac arrest is a leading cause of death among men not only in Indian sub continent but also in the entire world. MI or better known as Myocardial Infarction is becoming common in day to day life resulting loss of life. The problem mostly seen in men than in women, though the case of female is steeply growing. The World Health Organisation or WHO says almost 20 million people die of CAB causing a major concern for the health organisation to curb this menace. Coronary Artery Disease (CAD) is being treated as one of the most challenging disease in today's era. Almost seventy million Indians have CAD and thirty million have had a Myocardial Infarction (MI). CAD happens because of atherosclerotic occlusion of the coronary arteries. Half of all deaths basically are due to Cardio Vascular Disease (CVD).

Hence, there is an immediate requirement to look into this issue. The present research paper is a humble attempt to analyse and interpret the basic cause and the early detection of CAB (Coronary Artery Blockage). The blockages mostly due to some waxy material know as 'Atherosclerotic' plaque. In the present work attempts have been made to analyse and interpret the plaque formation area via soft computing techniques. The coronary blood flow highly relies on perfusion pressure and vessel resistance .The mathematical representation of Coronary Blood Flow (CBF) can be represented as follows:-

$\mathrm{CBF} \propto$ Perfusion Pressure (PP)...(i)

$\mathrm{CBF} \infty 1 /$ Vessel Resistance (VR)... (ii)

Coronary Perfusion (CP) is directly linked with diastole, so diastolic pressure is important. Coronary artery loses the power to release the vascular materials which allow the growing of $\mathrm{CP}$ as the demand increases. Various aspects of myocardial oxygen consumption are Contractility, Heart rate, Ventricular Wall Stress (VWS) to name a few.The most important one is the Heart rate. The stress on Ventricular wall is defined by Laplace's Law. The mathematical representation of the above can be given as follows:-

VWS $=[$ Left Ventricular Systolic Pressure (LVSP) * radius of Left Ventricle (LV)] /wall thickness ... (iii) As the number of patients with heart disease increases very rapidly, so there is a great need to develop radiologic tools for non-invasive imaging of the coronary arteries. Today, MRI, computed tomography (CT) techniques are available with high speed and spatial resolution with sophisticated electrocardiographic (ECG) synchronization and robustness of use. According to the various authors, this technique gives very good result in coronary artery stenosis but cannot be used for routine diagnostic. For this reason, some sophisticated techniques are required for precise diagnosis of CAD.

The paper has been organised as follows: - The literature review takes few of the salient work done in these area all around with the image processing perspective. With this the research gaps have been identified from which the objectives of the research work have been formulated. Later, the research methodology describes the entire process starting from the image acquisition to final ROI of the image. The experimental work has been done and the results have been discussed at a glance. The paper after giving the promising results concludes with marks on future enhancements and references taken. Needless to mention, the paper acknowledges all the hospitals/diagnostic centres and the doctors with patients who without any 'conflict of interest' provides the real (primary) data to pursue the current research work. 


\section{Literature Review}

As the number of patients with heart disease increases very rapidly ,so there is a great need to develop radiologic tools for non-invasive imaging of the coronary arteries. Now a days computed tomographic (CT) techniques are available with high speed and spatial resolution with sophisticated electrocardiographic synchronization and robustness of use. According to the authors this technique gives very good result in coronary artery stenosis but cannot be used for routine diagnostic.

International Journal of Computer Science \& Information Technology (IJCSIT), Vol. 2, No. 1, Serials Publications, New Delhi, 2009 An Effect of Spatial Filtering in Visualization of Coronary Arteries Imaging.Dr. P.S. Hiremath1, Mr. Kodge B.G.2 1. Professor \& Chairman, Department of Computer Sci. Gulbarga University, Gulbarga. State: Karnataka (INDIA) hiremathps@ hotmail.com 2. Lecturer, Department of Computer Science, S. V. College, Udgir - 413517, Dist. Latur State: Maharashtra (INDIA)

In today's world, we use coronary angiography for the detection of problem in coronary artery. In the conventional coronary angiography the procedure is no doubt invasive but has small risk of myocardial infections, stroke and death. On the other hand different non-invasive tools such as electrocardiography, echocardiography, and nuclear imaging are also used by cardiologists. These tools also have limitations in visualizing and quantifying coronary artery stenosis and predict the stability of plaques. One another noninvasive visualization technique for coronary arteries is available which is known as Coronary magnetic resonance angiography (MRA).This tool is in a developing stage with limitations and cannot be used for routine clinical practice. if research is done on improving the diagnostic resolution and accuracy of coronary MRA then it will give better results as compare to other existing tools. This paper will helps to cardiologists to take the clear look of spatial filtered imaging of coronary arteries.

Volume 3, Issue 8, August 2013 ,ISSN: 2277 128X International Journal of Advanced Researchin Computer Science and Software Engineering Research Paper Available online at: www.ijarcsse.com Calcification Detection in Coronary Arteries Using Image Processing Pankaj Goyal*KuldeepGoyalVipin Gupta Department of Electrical Engineering,Scientist, DIPR, Defence R \& D Senior Scientist, Philips DCRUST Murthal,Organization (DRDO), Research, ManyataTechSonepat, India Delhi, India Bangalore, India This technique only highlight the regions containing coronary arteries by using vessel enhancement diffusion filter in to the two dimensional DICOM (Digital imaging and Communications in Medicine) images taken from the 64-slice Computed Tomography Scan data of the heart. As in this angiography we are using 64-slice CT scan machine so we get better visualization of coronary arteries using a small dose of contrast .This was not possible with early techniques.Standardized evaluation methodology and reference database for evaluating coronary artery centerline extraction algorithms Michiel Schaapa, et.al.Authors have used CTA for reliable data in clinical practice. Various methods where used but no standard methodology has been published for the evaluation and comparison for various extraction algorithms used in coronary artery .The authors have used four fold mechanisms for establishing the bench mark for the extraction algorithms.

\section{Objectives:-}

1. Fuzzy quantification of various non-invasive imaging modalities related to CAB.

2. To propose 'if-then' rule based on various image processing tasks particularly used for Cardiac imaging.

3. To evaluate the ROI correspondence processes involved in the detection of blockage found in coronary arteries.

\section{Research Methodology}

Step-1 Undergone to the depth of heart anatomy and its physiology to know about various heart diseases and its consequences particularly taking the case of CAB.

Step-2 Unfolded all the possible parameters which are responsible for CAD.

Step-2.1 Investigated quantitatively all the factors related to CAD (Fuzzy Modelling).

Step-2.2 Inclusion of fuzzy logic and enhancing the present model to fuzzy based model for better blockage detection for realistic results.

Step-3 The input image is pre-processed and normalised for the further processing.

Step-4 After the normalization of the images, it undergoes for feature extraction using proposed fuzzy inference systems methodologies.

Step-5 Membership function has been generated for the real time values of the images.

Step-6 ROI is being analyzed and processed by using the proposed Fuzzy inference technique.

Step-7 Automated systems results were being validated with the actual output of the cardio images. 


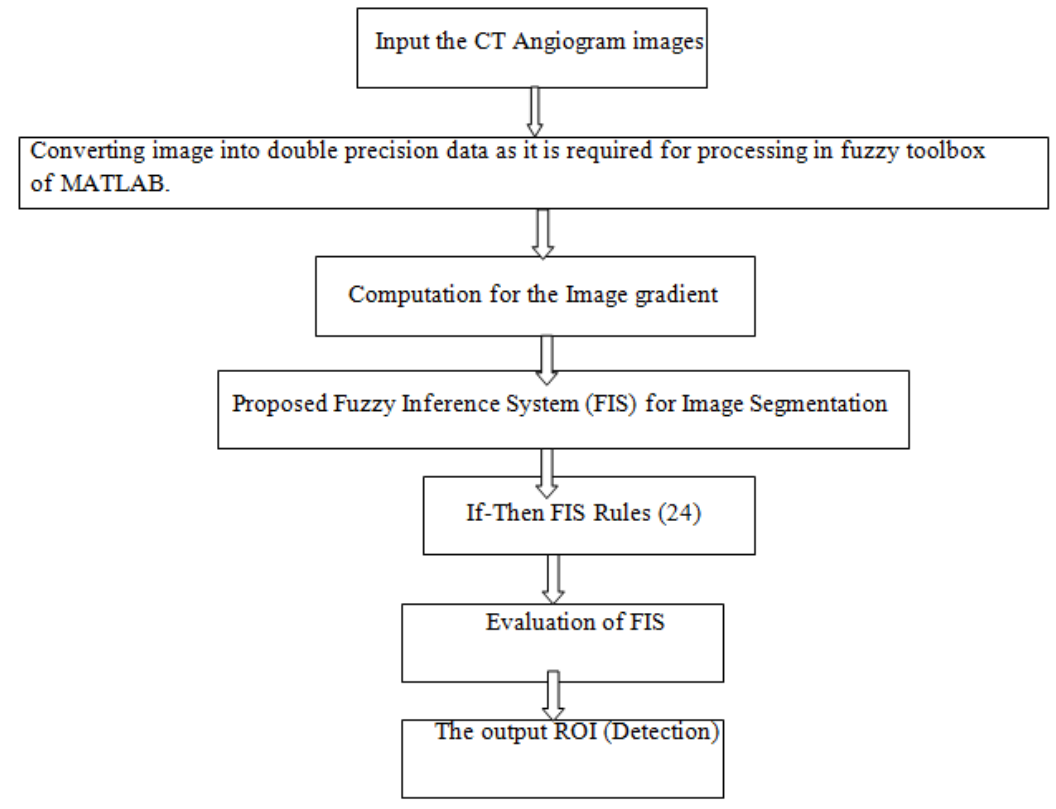

\section{CT Angiogram images}

Around 580 CT Angiogram Images (DICOM) have been taken for the current study. It has been divided into male and female with normal and healthy heart (CA in particular). The age group have been further divided into 35-65 yrs for female and 40-70 yrs for male. The background of every patient has also played an important factor in our study with their vivid living styles.

\section{Experimental Setup:-}

The present research has been carried out using MATLAB R2013b taking 32-bit Windows 7 professional SP1 as operating system. The processor used is Intel ${ }^{\circledR}$ Core $^{\mathrm{TM}} 2$ Duo CPU T6570 @2.10 GHz, RAM 2 GB. The primary data has been taken from Fortis hospital, H.P in DICOM image format. Sample of 580 images of the various stages of calcified images of CA has been considered and the same number of sample has been taken for the healthy patients. The age group taken in the primary sample source is in the range of 65-85 yrs and sex considered is both Male and Female.

\section{Results and Discussions}

The following image is a DICOM Image of a female heart taken by a snap shot of CORO 4/50 frame shot.

Figure 1: 4/50 frame short of Coronary Artery DICOM Image (Primary data set)

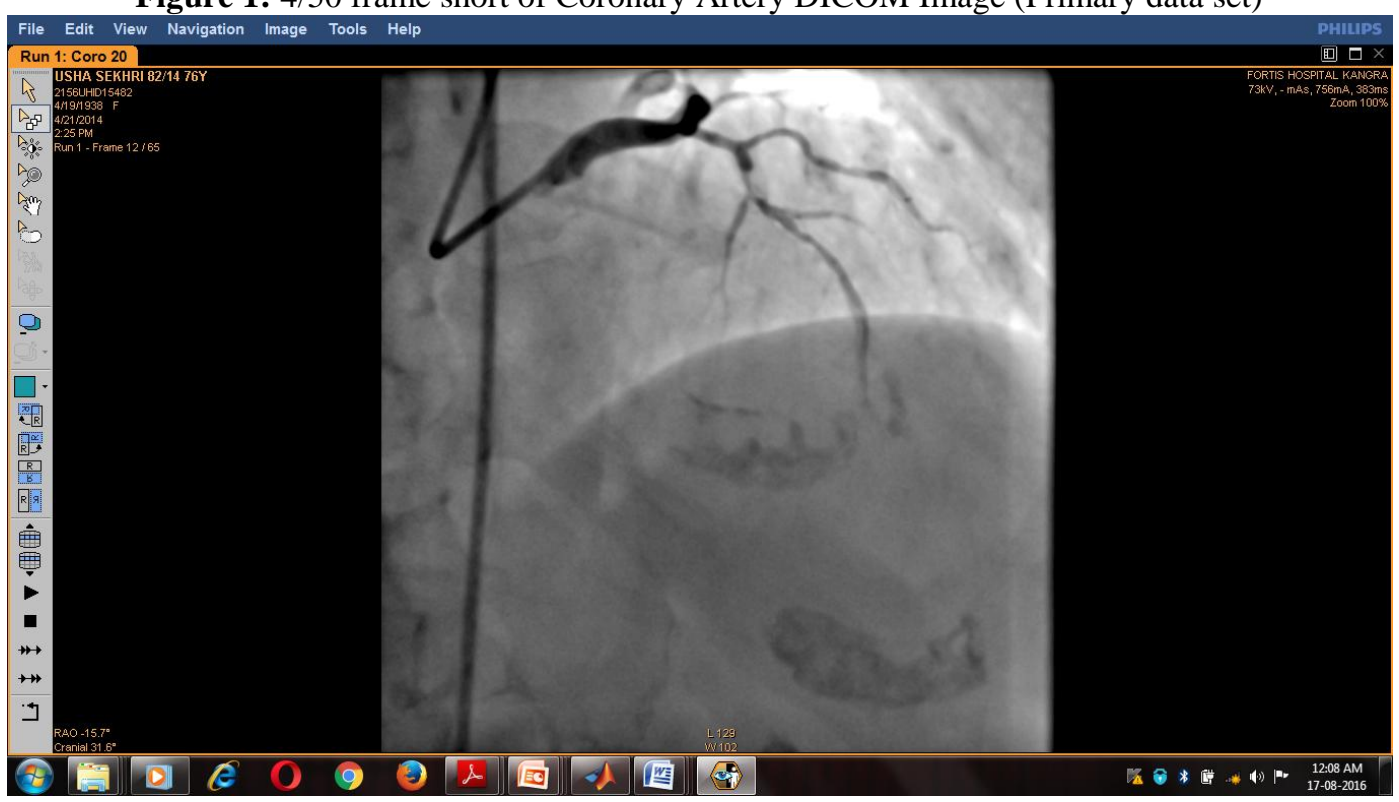


Figure 2: Matrix of 25 frame shots of 4/50 frame short of Coronary Artery DICOM Image (Primary data set)

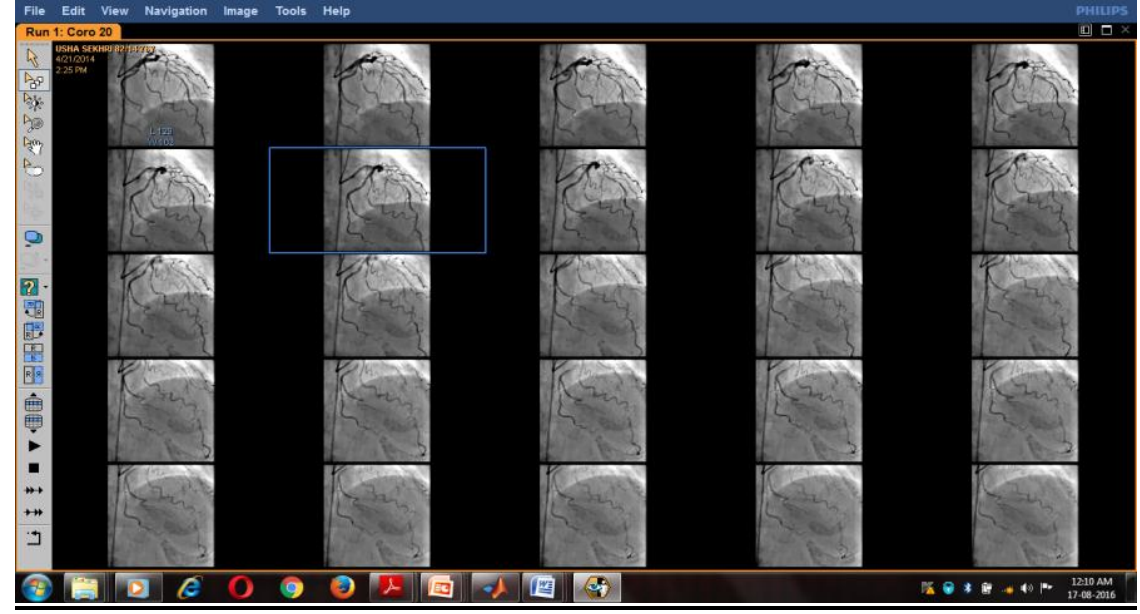

\section{Mathematical perspective of image segmentation technique Used:-}

Image segmentation plays a pivotal role for the analysis of any image. The region Extraction may be defined as a image I being divided into various regions say $r$ in the spatial domain and

is given as $\bigcup_{i=1} r i$

. Also, $r_{i} \cap r_{j}=\varnothing$. Images as taken undergone feature thresholding. The images taken has two types of regions and their distinctiveness of the segmented parts is evident by their feature values of all the pixels that belong to the regions of the one type having the value less than and equals to say ' $\mathrm{T}$ ' and the second type with of values having greater than or equals to ' $T$ '. This concept of hard thresholding pays a way to separate two regions with their hard threshold values and mathematically be given as

$$
\begin{gathered}
\mathrm{M}(\mathrm{r}, \mathrm{c})=\left\{\begin{array}{c}
1, \text { if } \mathrm{O}(\mathrm{r}, \mathrm{c})<=\mathrm{T} \\
0, \text { if } \mathrm{O}(\mathrm{r}, \mathrm{c})>\mathrm{T}
\end{array}\right\}
\end{gathered}
$$

Likewise, to get an optimal thresholding we may use the following taking two regions R1 and R2 with average graylevels say A1 and A2. The standard deviations are D1 and D2. The p.d.f are given by

$$
\begin{aligned}
& \mathrm{P} 1(\mathrm{z})=\frac{1}{\sqrt{2 \Pi D 1}} \exp \llbracket \frac{-(z-A 1)(z-A 1)}{2 D 1 * D 1} \rrbracket \\
& \mathrm{P} 2(\mathrm{z})=\frac{1}{\sqrt{2 \Pi D 2}} \exp \llbracket\left[\frac{-(z-A 2)(z-A 2)}{2 D 2 * D 2} \rrbracket\right.
\end{aligned}
$$

After applying the above mathematical model to the selected image, we get the following

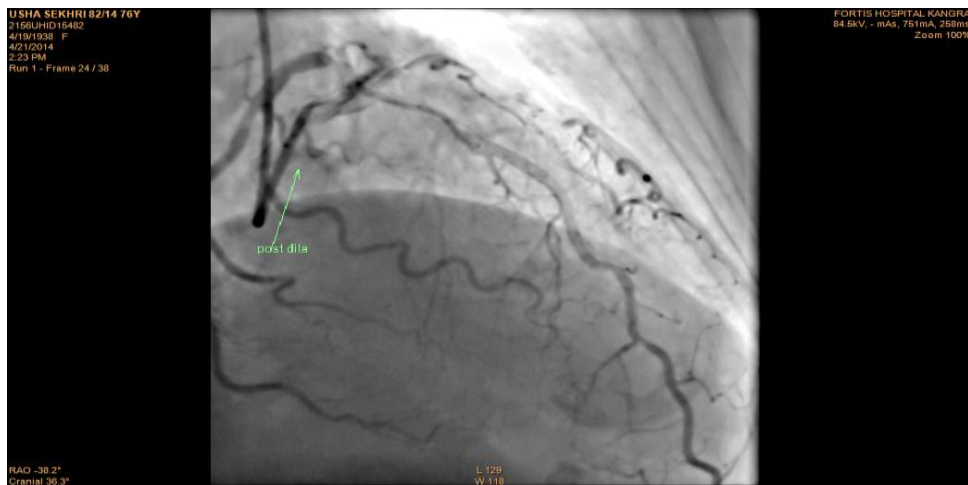

Figure 3: The simulated version of the mathematical perspective of Coronary Artery DICOM Image (Primary data set)

FIS for the four pixel matrix to extract the segmented ROI: - In the following case four pixel matrixes have been proposed in FIS for the computation of ROI using mamdani type. This is the high level display. The four inputs have been taken on the left and on the right the segmented region of interest (SEGROI). The data set have been loaded from the existing pixel data set and the matrixes have been formed. 


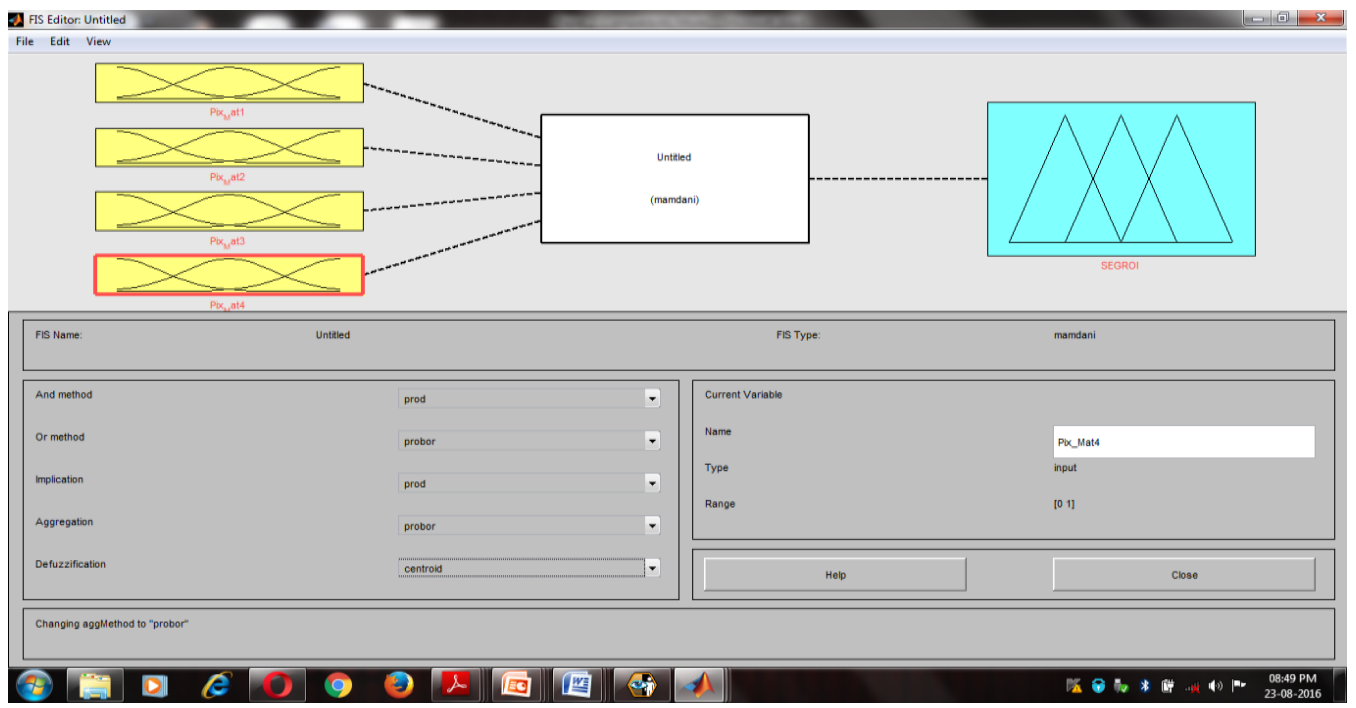

Figure 4(i): Four input and one output FIS for the fuzzy proposal

As stated earlier there were 24 rule base (if-then) rules included in the present research work.

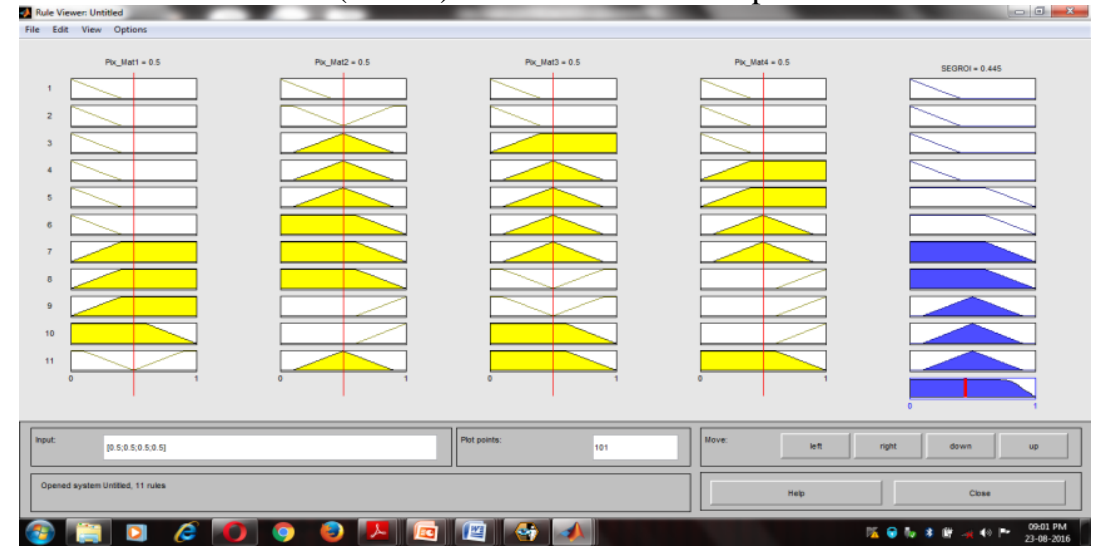

Figure 4 (ii) The snap shot of 11 rules have been shown in the results.

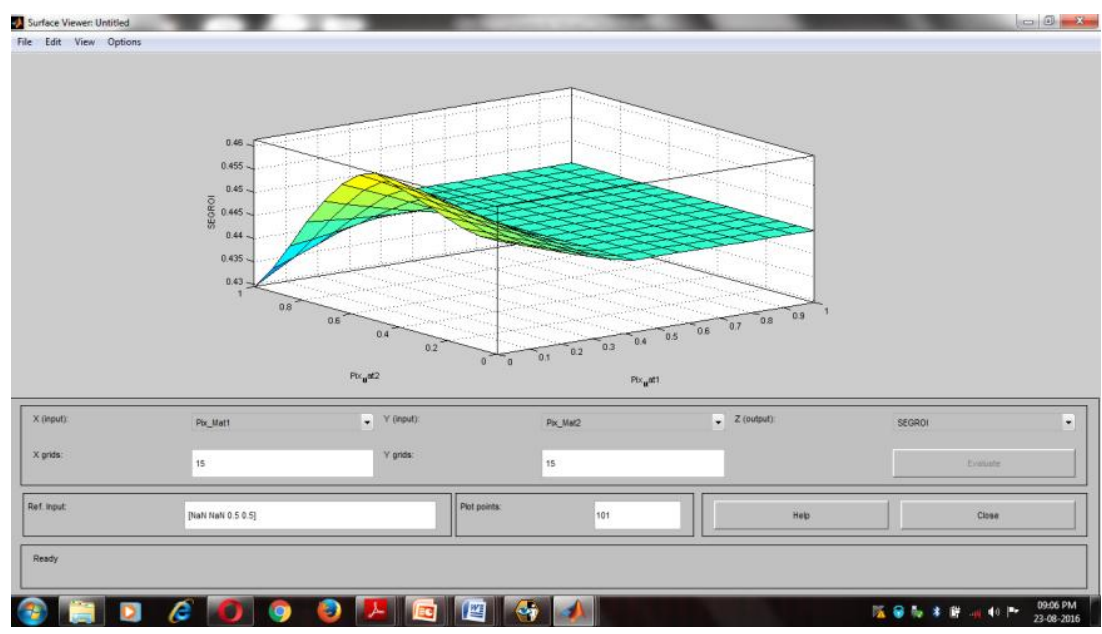

Figure: 5 -The surface viewer of the proposed 24 rules of FIS

The features of the above figure have been computed using GLCM.

Contrast: 7.4268

Correlation: 0.3346

Energy: 0.1623

Homogeneity: 0.3541

DICOM Data points extracts are as follows:- 
Analysis and Interpretation of Coronary Artery Blockage using Soft Computing Techniques

\begin{tabular}{|l|l|l|}
\hline$(0008,0005)$ & $\begin{array}{l}\text { Specific Character } \\
\text { Set }\end{array}$ & ISO_IR 100 \\
\hline$(0008,0008)$ & Image Type & DERIVED, PRIMARY, SINGLE PLANE, SINGLE A \\
\hline$(0008,0016)$ & SOP Class UID & 1.2 .840 .10008 .5 .1 .4 .1 .1 .12 .1 \\
\hline$(0008,0018)$ & SOP Instance UID & 1.3 .12 .2 .1107 .5 .4 .5 .135181 .30000014040803282953100000027 .512 \\
\hline$(0028,1050)$ & Level Image & 129 \\
\hline$(0028,2110)$ & $\begin{array}{l}\text { Lossy } \\
\text { Compression }\end{array}$ & Siemens \\
\hline$(0008,0070)$ & Manufacturer & $\begin{array}{l}\text { Manufacturer's } \\
\text { Model Name }\end{array}$ \\
\hline$(0008,1090)$ &
\end{tabular}

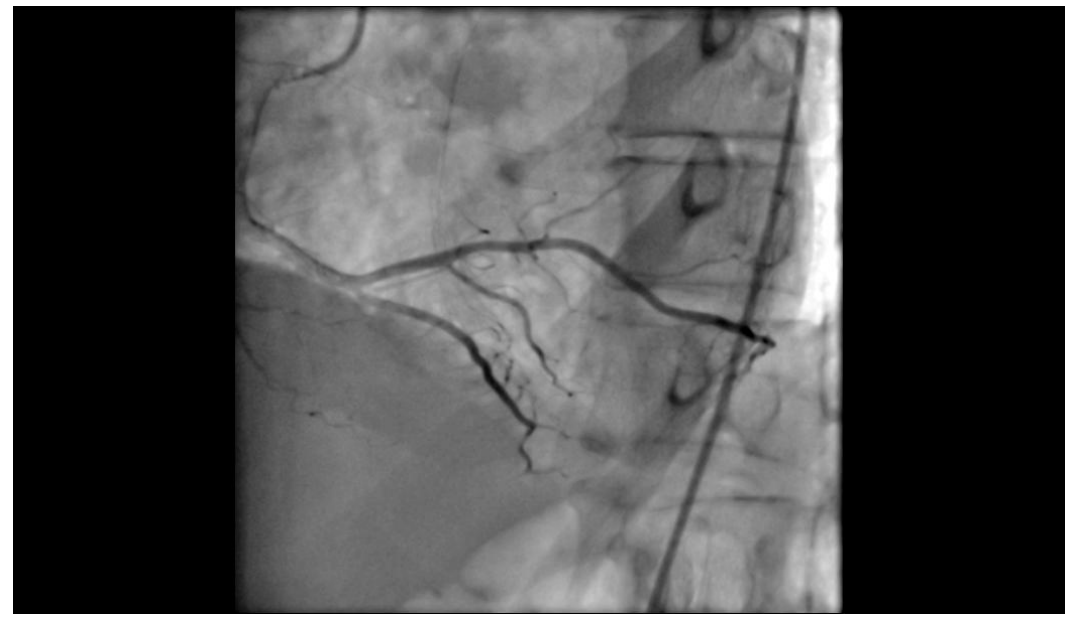

Figure 6 (i) Segmentation of the ROI using the above FIS

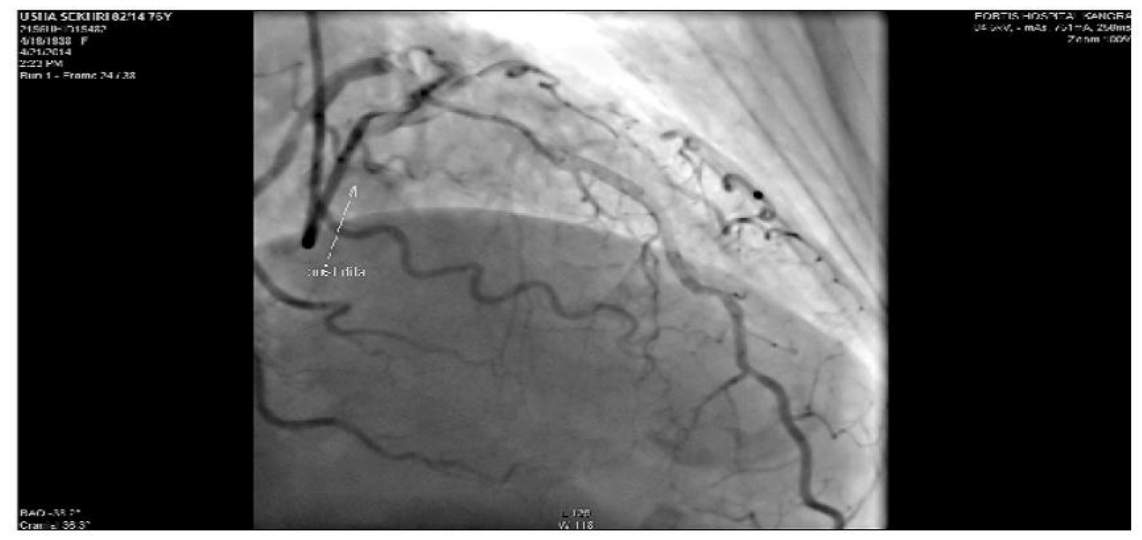

Figure 6 (ii) Segmentation of the enhanced images for ROI using the above FIS

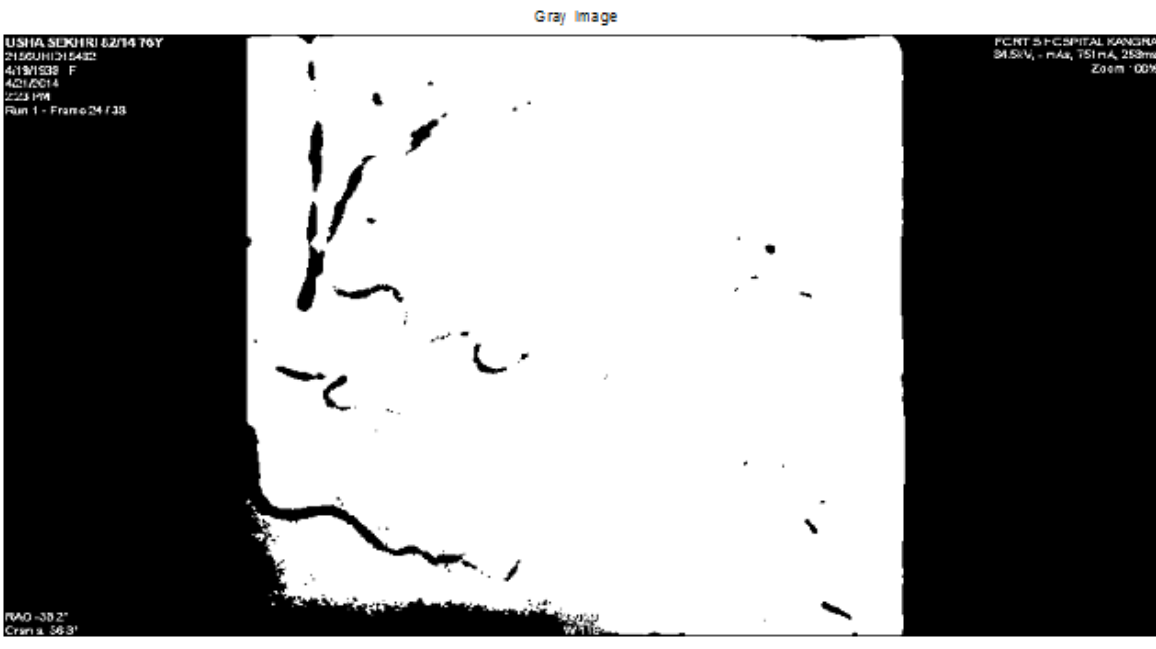

Figure7. The following is the output of fuzzy logic inclusion with 24 if-then 

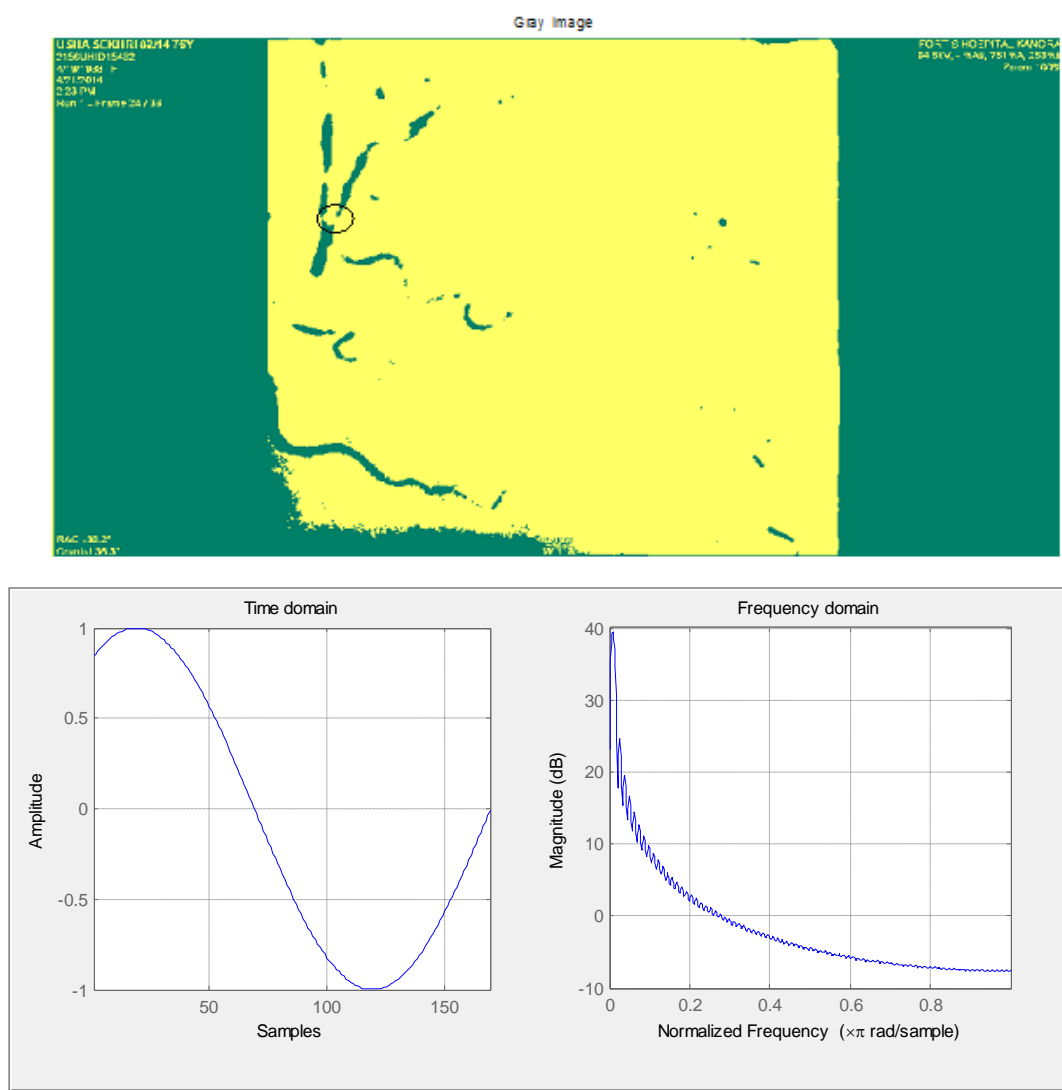

Figure 8: Time and Frequency domain correspondence of the above coronary blockage

\section{Conclusion}

The present research is a boost for cardiac Imaging researcher in the way that better results have been obtained using soft computing technique. We have investigated every parameter related to cardiac non-invasive imaging modalities. The image processing aspects have been dealt in accordance with the need of the cardiologist . The final output is promising for the early detection of CAD.

\section{References}

[1]. Koen Nieman ,'International Task Force for Prevention of Coronary Heart Diseas- MSCT in coronary heart disease," Rotterdam Feb 2003

[2]. Leo P. Lawler; Harpreet K. Pannu; Elliot K. Fishman Am J Roentgenol,'MDCT Evaluation of the Coronary Arteries, 2004: How We Do It-Data Acquisition, Postprocessing, Display, and Interpretation ," 2005; 184 (5): 1402-1412. C2005 American Roentgen Ray Society

[3]. H. Mahnken, Georg Mühlenbruch, Guido Dohmen, MalteKelm, Joachim E. Wildberger, Rolf W. Günther,’Review article on Current State of Non-Invasive Coronary MSCT Angiography," Andreas-Technical note.

[4]. Al Moudi M1, Sun Z*,1, Lenzo N2,'Biomedical Imaging and Intervention Journal REVIEW ARTICLE ,Diagnostic value of SPECT, PET and PET/CT in the diagnosis of coronary artery disease: A systematic review,"Received 18 June 2010 ; received in revised form 5 October 2010; accepted 9 October 2010

[5]. U. Joseph Schoepf, MD Christoph R. Becker, MD Bernd M. Ohnesorge, PhD E. Kent Yucel, MD,’CT of Coronary Artery Disease,"Published online 10.1148/radiol.2321030636 Radiology 2004; 232:18-37,Volume 232 Number 1 CT of Coronary Artery Disease

[6]. Dr. P.S. Hiremath1, Mr. Kodge B.G," An Effect of Spatial Filtering in Visualization of Coronary Arteries Imaging,'International Journal of Computer Science \& Information Technology (IJCSIT), Vol. 2, No. 1, Serials Publications, New Delhi, 2009.

[7]. Pankaj Goyal,KuldeepGoyalVipin Gupta ," on International Journal of Advanced Research in Computer Science and Software Engineering Calcification Detection in Coronary Arteries Using Image Processing,"Volume 3, Issue 8, August 2013 ,ISSN: 2277 $128 \mathrm{X}$

[8]. Michiel Schaapaet.al"Standardized evaluation methodology and reference database for evaluating coronary artery centerline extraction algorithms"-Technical Note.

[9]. Labadin.J, Ahmadi.A (2006) Mathematical Modeling of the Arterial Blood Flow Mathematical Modeling of the Arterial Blood Flow, Proceedings of the 2nd IMT-GT Regional Conference on Mathematics, Statistics and Applications, Universiti Sains Malaysia, Penang

[10]. Fibich.G, Lanir.Y, Liron.N (1993) Mathematical model of blood flow in a coronary capillary, Am. J. Physiol. 265 (Heart Circ. Physiol. 34): H1829-H1840

[11]. Diagnosing coronary artery disease by sound analysis from coronary stenosis induced turbulent blood flow: diagnostic performance in patients with stable angina pectoris Simon Winther, et.al (2015), Int. Journal of Cardiovascular Imaging,Springer Pub (DOI 10.1007/s10554-015-0753-4). 